\title{
Cognición y autonomía en personas mayores dependientes
}

\author{
Joaquín Álvarez-Hernández, José Manuel Aguilar-Parra* e Isabel Mercader-Rubio
}

Universidad de Almería

\begin{abstract}
Resumen: El objetivo de este trabajo ha sido relacionar el deterioro cognitivo en las personas mayores con la conservación de aquellas actividades de la vida cotidiana que resultan más complejas y que les proporcionan un estado de autonomía avanzado. La muestra ha estado compuesta por 472 pacientes (163 varones y 309 mujeres), con edades comprendidas entre 60 y 102 años. El diseño se corresponde con un estudio descriptivo correlacional de investigación evaluativa. Resultados. Se analizan los resultados obtenidos a través de la contestación a los instrumentos de evaluación SPMQ (Pfeiffer) y ESENP, contemplando el estado mental de los pacientes y comprobando el manejo de estos en cuanto a la realización de actividades de la vida diaria, así como la relación entre ambos factores teniendo en cuenta además si existen diferencias en función de las edades y el género. Conclusiones. Los datos registrados de este estudio muestran la relación que existe entre el deterioro cognitivo y la autonomía personal en personas mayores lo que permite definir con precisión qué necesidades reales demanda la población futura en cuanto a servicios sociales comunitarios, pudiéndolos utilizar con la intención de optimizar y ampliar dichos servicios.

Palabras clave: Cognición; autonomía personal; deterioro cognitivo; personas mayores.
\end{abstract}

\section{Introducción}

El límite entre el envejecimiento normal y el envejecimiento patológico recibe cada vez más atención por parte de la literatura especializada según apuntan Calero y Navarro (2003). De esta forma, la vejez parece llevar unida un incremento en la probabilidad de sufrir determinadas enfermedades así como experimentar un relativo declive intelectual.

Paúl, Ribeiro y Santos (2010) afirman que el deterioro cognitivo es uno de los temas más relevantes en los estudios clínicos del envejecimiento. La asociación entre el aumento de envejecimiento de la población y las enfermedades neurológicas de gran alcance, ha dado lugar a una alta prevalencia de deterioro cognitivo en las personas mayores, especialmente en las más ancianas (Arthanat, Nochajski y Stone, 2004; St John, Montgomery, Kristjansson y McDowell, 2002). Por lo menos, el $10 \%$ de las personas mayores de 65 años y el $50 \%$ de los mayores de 85 años tienen algún tipo de deterioro cognitivo, que van desde los déficits leves a graves (Jorm y Jolley, 1998). Basado en una revisión sistemática de deterioro cognitivo en la población general población de edad avanzada, Park, O'Connell y Thomson (2003) muestran que un cierto grado de deterioro cognitivo es casi universal y se puede esperar en la mayoría de las personas más ancianas, y que la prevalencia de deterioro cognitivo y la tasa de decadencia aumentan con la edad.

Según Calero y Navarro (2003) el deterioro cognitivo leve, se refiere al estado en el que una función cognitiva se de-

* Dirección para correspondencia [Correspondence address]: José Manuel Aguilar Parra. Universidad de Almería. Departamento de Psicología Evolutiva y de la Educación. La Cañada de San Urbano, s/n 04120 Almería (España).E-mail: jmaguilar@ual.es
Title: Cognition and autonomy in dependent elderly people.

Abstract: The aim of this work has been to relate the cognitive impairment in elderly people to the preservation of those daily life activities that turn out to be more complex and that provide them with a state of advanced autonomy. The sample has been composed of 472 patients (163 males and 309 women), at ages understood between 60 and 102 years. The design corresponds to the correlational descriptive study of evaluative investigation. Results. The results obtained have been analyzed though the answers to the instruments of evaluation SPMQ (Pfeiffer) and ESENP, contemplating the mental condition of the patients and verifying their managing of the daily life activities, as well as the relation between both factors taking into account differences depending on the ages and sexes. Conclusions. The information registered in this study shows us the relation that exists between cognitive deterioration and the personal autonomy in elderly people what allows to define accurately the real needs of the future population as for social community services, being able to use them with the intention of optimizing and extending the above mentioned services.

Key words: Cognition; personal autonomy; cognitive impairment; elderly.

teriora a un nivel mayor de lo que se anticiparía por la edad de la persona, aunque ésta todavía no cumpla los criterios de demencia y funcione de forma independiente en la vida diaria. Werner y Korczyn (2008) lo definen como una condición caracterizada por el declive cognitivo adquirido recientemente en una medida que está más allá de lo esperado para su edad o nivel educativo, sin embargo, no causan un deterioro funcional importante.

Se considera que la evaluación del funcionamiento intelectual durante la vejez es uno de los principales objetivos, puesto que va asociado con otros padecimientos físicos y conductuales. Así, evaluar el funcionamiento intelectual está presente con frecuencia en toda evaluación psicológica de las personas mayores.

Tomando como referencia a Fontán, Lorenzo y Silveira (2004), se puede afirmar que el diagnóstico del deterioro cognitivo se basa en los siguientes criterios:

1) Queja subjetiva de déficit de memoria, preferentemente corroborado por un familiar.

2) Déficit memorístico objetivable mediante test.

3) Ausencia de otros elementos de deterioro.

4) Ausencia de demencia.

5) Actividades de la vida diaria conservadas.

Esta investigación se centra en plantear y estudiar cómo hay elementos que determinan el deterioro en la vejez a través de las actividades de la vida diaria conservadas, relacionándolas con edad y sexo de los sujetos, teniendo en cuenta el estado mental cognitivo en el que se encuentran. Se ha de destacar que Álvarez y Sicilia (2007) realizaron una investigación similar precedente a esta, en la que se determinó la relación entre el deterioro cognitivo y la autonomía personal básica en personas mayores. Ahora, se pretende va- 
lorar también actividades de la vida diaria, pero a un nivel más avanzado, por ejemplo: planchar, usar transportes públicos, etc., en contraste con la de los autores señalados anteriormente, que trabajaron sobre actividades de la vida diaria básicas como vestirse, lavarse, comer, etc.

Precede a esta investigación la realizada por Wadley et al. (2007) en la que se muestra la importancia del estudio del deterioro cognitivo en relación con las actividades de la vida diaria y la pérdida de funcionalidad en las mismas, siendo éstas, claves para la autonomía y la calidad de vida de la persona.

Bowling (1994) define el estado funcional del anciano como el grado en que un individuo es capaz de desarrollar los papeles que tiene asignados libre de limitaciones físicas y/o mentales.

Para autores como Schuz, Heckausen y O’Brien (1994), la mayoría de los individuos ancianos experimentan declives funcionales en dominios o situaciones de la vida bastante importantes. Estos declives disminuyen la habilidad para permanecer independiente en la comunidad y comprometen seriamente la calidad de vida.

Neugarten (1982) señala que la autonomía funcional disminuye progresivamente en el curso del envejecimiento. Este descenso de la autonomía funcional incide sobre diferentes aspectos de la vida, sobre diferentes matices psicológicos y en particular sobre el futuro personal, tal y como afirman Bouffard y Bastin (1994).

Así, además de evaluar la salud física en las personas mayores hay que hacer análisis y concreción de las Actividades de la Vida Diaria (A.V.D.), englobando tanto actividades instrumentales de cuidado personal y actividades instrumentales de índole doméstico, siendo las más complejas las de vida independiente.

Baltes, Mayr, Borchelt, Maas y Wilms (1993), han podido demostrar cómo el grado de competencia de la vida diaria influye en la independencia de una persona y en el dominio acerca de las actividades de la vida diaria.

Las Creencias culturales de la sociedad actual, sobre la importancia de la autonomía personal, puede provocar un descenso de la autoestima y un impacto negativo en aquellos mayores que por pérdidas de salud se han visto obligados a depender de otras personas para realizar las actividades cotidianas de la vida diaria, tal y como apuntan Baltes y WernerWahl (1990).

La relevancia del estado funcional la resalta la Organización Mundial de la Salud, OMS, cuando manifiesta que «como mejor se mide la salud en el anciano es en términos de función», haciendo referencia a que las medidas generales de salud poseen un valor limitado para indicar el grado de independencia que puede conseguir un individuo a pesar de la enfermedad (Martín, 1998, citado en Bernal y Barbero, 2006).

No es suficiente enumerar, conocer y tratar de forma aislada los problemas médicos que presenta el anciano, sino que es necesario considerar cómo esos problemas afectan a la capacidad funcional del individuo. Por ello, parece preciso considerar el concepto A.V.D. (Actividades de la Vida Diaria).

Al revisar diversas escalas de actividades instrumentales de la vida diaria se encuentra que todos los elementos utilizados en ellas pueden integrarse en las siguientes diez dimensiones:

1) Cocinar.

2) Comprar.

3) Limpiar.

4) Lavar / planchar.

5) Tomar medicamentos.

6) Llevar contabilidad.

7) Usar teléfono.

8) Uso del transporte público.

9) Subir / bajar escaleras.

10)Andar / pasear.

La importancia de la valoración social de los ancianos viene dada por el funcionamiento social que se correlaciona con el funcionamiento físico y mental. Así, el bienestar social mejora la capacidad de manejar problemas de salud y persevera la autonomía, a pesar de las limitaciones funcionales.

Black y Rush (2002) concluyen que el deterioro cognitivo $\mathrm{y}$ funcional parecen influirse mutuamente, es decir, el deterioro de un área, ya sea la cognitiva o la funcional, va a influir en el deterioro de la otra de forma significativa. Estos mismos autores, señalan que el conocimiento de las condiciones de salud crónicas específicas que aumentan el riesgo de deterioro cognitivo o funcional en diferentes grupos étnicos, y el efecto de las enfermedades concomitantes, también puede ayudar en los esfuerzos para prevenir el deterioro en personas mayores.

En este sentido, se plantean como propósitos de esta investigación los siguientes objetivos:

- Conocer qué componentes de la autonomía personal correspondientes a las AIVD (cocinar, comprar, limpiar, lavar / planchar, tomar medicamentos, llevar contabilidad, usar teléfono, uso el transporte público, subir / bajar escaleras, andar / pasear) forman parte del repertorio de los usuarios de la muestra que conforman este estudio y comprobar si existen diferentes en función del sexo y edad.

- Conocer la relación entre el estado mental de los sujetos de la muestra y el factor de autonomía referente a las AIVD.

A través de instrumentos como el Cuestionario de examen cognitivo SPMSQ (Pfeiffer) se ha medido el estado mental cognitivo de los sujetos, y la Escala de Evaluación de Necesidades Psicosociosanitarias (ESENP) para medir estas variables que interesaban para este estudio. 


\section{Método}

\section{Participantes}

Los sujetos que componen la muestra objeto de estudio han sido 472, 163 varones y 309 mujeres, de edades comprendidas entre 60 y 102 años, residentes en la provincia de Almería, tal y como se puede apreciar en la Tabla 1. La muestra abarca la población total de usuarios de Servicios de Ayuda a Domicilio (SAD) de la provincia de Almería que cubren los Servicios Sociales de la Diputación de Almería. Del total de usarios del SAD, se realizó un muestreo por conglomerados, determinando al azar los municipios y sujetos a los que se encuestaría posteriormente.

Tabla 1. Distribución de la muestra por edad y sexo.

\begin{tabular}{lllll}
\hline Edad & Hombres & Mujeres & Total & Porcentajes \\
\hline $60 / 69$ años & 39 & 44 & 83 & $17.6 \%$ \\
$70 / 79$ años & 84 & 142 & 226 & $47.6 \%$ \\
$80 / 89$ años & 35 & 99 & 134 & $28.7 \%$ \\
+ de 90 años & 5 & 24 & 29 & $6.2 \%$ \\
Total & 163 & 309 & 472 & $100 \%$ \\
\hline
\end{tabular}

Como muestra la Tabla 1, el grueso de la población se encuentra en las edades comprendidas entre 70/79 años, seguidos del grupo edad 80/89 años.

\section{Instrumentos de evaluación}

Para cubrir el objetivo principal de esta investigación, se utilizó el SPSMQ para la medida del estado mental y se elaboró “ad hoc" la Escala de Evaluación de Necesidades Psicosanitarias.

El "Short Portable Mental Status Questionnaire de Pfeiffer” (SPSMQ, 1975) se desarrolló a partir de la Escala de Memoria de Wechsler (Wechsler, 1945). Forma parte del instrumento multidimensional OARS (Olders Americans Resources and Services Questionnaire, Duke University, 1978), aunque se puede administrar de manera independiente al resto del instrumento.

Según Del Ser Quijano y Peña-Casanova (1994) el SPMSQ es considerado actualmente uno de los tests de rastreo más adecuado para su aplicación en poblaciones generales, porque muestra una fiabilidad bastante adecuada, presentando unos índices de correlación test-retest entre 0.82 y 0.83 .

El SPMSQ consta de diez elementos que valoran la memoria, la orientación y la capacidad de ejecutar tareas seriales aritméticas, y permite, a través de la cuantificación del número de errores, establecer el nivel de estado mental cognitivo. En este estudio se utiliza la adaptación española realizada por la Cátedra de Psicodiagnóstico de la Universidad Autónoma de Madrid, (Fernández-Ballesteros, 1992).

Con base en la estandarización original y dependiendo del número de errores es posible clasificar al usuario como "intacto", con deterioro intelectual "leve", "moderado" y "severo",
La adaptación española, tras ser sometida a estudios de validación, se ajusta aceptablemente bien a las categorías de Pfeiffer (Fernández-Ballesteros, 1994). Para garantizar la calidad de los datos hemos estimado la fiabilidad mediante el modelo de dos mitades. Así, el modelo divide la escala y examina la correlación entre estas dos partes y como se puede apreciar, el coeficiente es lo suficientemente elevado para considerar aceptable el nivel de fiabilidad.

Correlation between forms $=.7840 \quad$ Equal-length Spearman-Brown $=.8789$

El segundo instrumento es la Escala de Evaluación de Necesidades Psicosociosanitarias (ESENP).

Este instrumento consta de 54 elementos integrados en un total de 8 dimensiones, junto con las variables sociodemográficas (sexo, edad, estado civil, estudios, etc.).

En cuanto al tipo de respuesta que tiene este cuestionario se encuentra que la escala de medida incluía formatos de respuesta de tres tipos: dicotómicas, categóricas y de opción múltiple. Para este estudio, se han utilizado únicamente ítems de respuestas categóricas en las cuales puede responder una de las tres posibilidades, por ejemplo "de las actividades que le nombro cuáles puede realizar, cuáles no y cuáles sí pero con dificultad" (Sí, No, Con Dificultad).

De todos los aspectos que con este cuestionario se evalúan, en esta investigación se ha atendido a la autonomía personal correspondiente a las AIVD, que se evalúa a través de las respuestas que dan los usuarios S.A.D. sobre conductas como: cocinar, comprar, limpiar, lavar/planchar, tomar medicamentos, llevar contabilidad, usar teléfono, uso del transporte público, subir/bajar escaleras, andar/pasear. La fiabilidad que presenta este cuestionario es $\alpha=.7377$.

\section{Procedimiento}

Se pretendió que estos mayores se sintieran más tranquilos y seguros contestando las preguntas a personas conocidas de su entorno para evitar las reticencias que pudieran presentar frente a preguntas más íntimas, por lo que la aplicación de los instrumentos se realizó por parte de los propios trabajadores de los centros de las zonas de la provincia de Almería, Trabajadores sociales, Psicólogos...), por lo que fue necesario iniciar contactos previos con ellos para garantizar su participación y facilitarles toda la información que fuera necesaria.

Se le encargó a cada técnico de zona la tarea de pasar unos 20-25 cuestionarios a los usuarios, dándoles un tiempo de 6 meses para la recogida, es decir 3-4 usuarios al mes, para que realizaran tranquilamente su labor y seleccionaran el momento más adecuado del usuario.

\section{Diseño}

El diseño se corresponde con un estudio descriptivo correlacional de investigación evaluativa para conocer y diagnosticar las dimensiones de una realidad concreta, valorarla y determinar una toma de decisiones. 
Para todos los análisis estadísticos se ha utilizado el $\mathrm{Pa}$ quete Estadístico para Ciencias Sociales (SPSS 18.0).

\section{Resultados}

Para el cálculo de los resultados, se puede señalar que los parámetros descriptivos fueron realizados mediante el análisis de frecuencias. Se utilizaron las tablas de contingencia y sus estadísticos asociados como son la prueba de chicuadrado para comprobar si entre las variables existía o no algún tipo de relación. Para cuantificar este grado de asociación entre las variables se utilizó el índice de asociación del coeficiente de contingencia y para poder interpretar con precisión el significado de la asociación detectada se usó el estadístico de los residuos tipificados corregidos

\section{Análisis de la Autonomía Personal referente a las AIVD}

El primer objetivo que se plantea "conocer qué componentes de la autonomía personal correspondientes a las AIVD (cocinar, comprar, limpiar, lavar / planchar, tomar medicamentos, llevar contabilidad, usar teléfono, uso el transporte público, subir / bajar escaleras, andar / pasear) forman parte del repertorio de los usuarios de la muestra y comprobar si existen diferentes en función del sexo y edad"

La autonomía personal correspondiente a las AIVD define las actividades de la vida diaria relacionadas con el ámbito doméstico que son necesarias para vivir. Según los estadísticos obtenidos de la muestra, reflejados en la Tabla 2, se puede afirmar que actividades como cocinar, tomarse los medicamentos, llevar la contabilidad, usar el teléfono, subir/bajar escaleras y andar y pasear son realizadas normalmente o con dificultad por una proporción mayor del $50 \%$ de la población muestral. Sin embargo, se encuentran actividades como comprar, limpiar, lavar o planchar y usar el transporte público que no son realizadas por más del 50\% de las personas que componen la muestra. La actividad más realizada sin problemas es tomarse sus medicamentos, se- guida de usar el teléfono y llevar la contabilidad, por el contrario, las que menos se realizan son limpiar, lavar/planchar y usar el transporte público.

Tabla 2. Porcentaje de dependencia que presentan las personas usuarias del SAD en relación a la realización de las AIVD.

\begin{tabular}{llll}
\hline Actividad & Sí la realiza & $\begin{array}{l}\text { La realiza con } \\
\text { dificultad }\end{array}$ & No la realiza \\
\hline Cocinar & $28.2 \%$ & $28.4 \%$ & $43.4 \%$ \\
Comprar & $23.0 \%$ & $24.8 \%$ & $52.2 \%$ \\
Limpiar & $2.20 \%$ & $18.5 \%$ & $79.3 \%$ \\
Lavar/planchar & $7.80 \%$ & $20.8 \%$ & $71.4 \%$ \\
Tomar medicamentos & $68.7 \%$ & $8.30 \%$ & $23.0 \%$ \\
Llevar la contabilidad & $50.8 \%$ & $12.6 \%$ & $36.6 \%$ \\
Usar el teléfono & $58.1 \%$ & $11.3 \%$ & $30.6 \%$ \\
Utilizar transporte & $21.4 \%$ & $16.7 \%$ & $62.0 \%$ \\
público & & $36.9 \%$ & $41.1 \%$ \\
Subir/bajar escaleras & $22.1 \%$ & $35.1 \%$ & $25.6 \%$ \\
Andar/pasear & $29.3 \%$ & $45 \%$ \\
\hline
\end{tabular}

La conclusión a la que se llega, a la vista de los resultados, es que en la mayoría de los casos, la mitad de la población, en algunos casos más, necesita ayuda para realizar las actividades propias de tipo doméstico como cocinar, limpiar la casa, lavar y planchar la ropa, etc.

Analizados los datos en función del sexo y la edad se obtienen los siguientes resultados:

\section{En función del sexo}

La significación de $p<.001$ permitió rechazar la hipótesis de independencia. Por lo tanto, se puede afirmar que existe relación entre ser hombre o mujer y la autonomía personal correspondiente para desarrollar las AIVD.

En la Tabla 3 se observa que las conductas que determinan el grado de autonomía correspondiente a las AIVD que se muestran relacionadas con la variable sexo son: comprar, lavar / planchar, llevar la contabilidad, uso del transporte público, subir y bajar escaleras y andar/pasearse.

Tabla 3. Relación entre ser hombre o mujer y la autonomía personal correspondiente a las AIVD.

\begin{tabular}{|c|c|c|c|c|c|c|c|c|c|c|}
\hline \multirow[t]{4}{*}{ CONDUCTA } & \multirow[t]{4}{*}{ Chi-cuadrado } & \multirow{4}{*}{$\begin{array}{l}\text { Valor coeficiente } \\
\text { de contingencia }\end{array}$} & \multirow[t]{4}{*}{ Sig } & \multirow{4}{*}{$\begin{array}{l}\text { Relacionada } \\
\text { Independiente }\end{array}$} & \multirow{2}{*}{\multicolumn{6}{|c|}{$\begin{array}{l}\text { Residuos tipificados corregidos } \\
\text { Nivel de capacidad por género }\end{array}$}} \\
\hline & & & & & & & & & & \\
\hline & & & & & \multicolumn{3}{|c|}{ Hombres } & \multicolumn{3}{|c|}{ Mujeres } \\
\hline & & & & & Sí & $\mathrm{CD}$ & No & Sí & $\mathrm{CD}$ & No \\
\hline Cocinar & .212 & .082 & .215 & $\mathrm{I}$ & .3 & -1.7 & 1.3 & -.3 & 1.7 & -1.3 \\
\hline Comprar & .000 & .295 & .000 & $\mathrm{R}$ & 6.5 & -1.6 & -4.0 & -6.5 & 1.6 & 4.0 \\
\hline Limpiar & .388 & .065 & .388 & I & .4 & 1.3 & -1.4 & -.4 & -1.3 & 1.4 \\
\hline Lavar/planchar & .043 & .118 & .043 & I & -1.8 & -1.4 & 2.4 & 1.8 & 1.4 & -2.4 \\
\hline Tomar medicamentos & .296 & .063 & .296 & I & .6 & -1.3 & .3 & -.6 & 1.3 & -.3 \\
\hline Llevar contabilidad & .016 & .151 & .016 & $\mathrm{R}$ & 2.9 & -.8 & -2.5 & -2.9 & .8 & 2.5 \\
\hline Usar teléfono & .656 & .037 & .656 & I & .6 & -.7 & -.2 & -.6 & .7 & .2 \\
\hline Tras. público & .000 & .347 & .000 & $\mathrm{R}$ & 7.1 & -1.2 & -5.1 & -7.1 & 1.2 & 5.1 \\
\hline Subir/bajar escaleras & .000 & .312 & .000 & $\mathrm{R}$ & 6.7 & -1.4 & -4.3 & -6.7 & 1.4 & 4.3 \\
\hline Andar/pasear & .000 & .283 & .000 & $\mathrm{R}$ & 6.1 & -4.1 & -1.7 & -6.1 & 4.1 & 1.7 \\
\hline
\end{tabular}

Nota: $\mathrm{Sí}=$ La realiza; $\mathrm{CD}=$ La realiza con dificultad; $\mathrm{No}=$ No la realiza. 
Según los resultados obtenidos se puede concluir que en todas las conductas hay más hombres de los esperados que mujeres que desarrollen estas conductas de forma autónoma. Únicamente la actividad de lavar y planchar aparece más realizada por las mujeres que por lo hombres.

\section{En función de la edad se concluye lo siguiente:}

La Significación de $p<.001$ permite rechazar la hipótesis de independencia. Y por lo tanto, es posible afirmar que existe relación entre la edad y la autonomía personal referente al desarrollo de las AIVD.

Se puede observar, tal y como se muestra en la Tabla 4 , que las conductas que determinan el grado de autonomía correspondiente a las AIVD que se muestran relacionadas con la variable edad son: cocinar, comprar, lavar / planchar, tomar medicamentos, llevar la contabilidad, uso del teléfono, uso del transporte público, subir y bajar escaleras y andar/pasearse. Obteniendo la puntuación más alta de relación el uso del teléfono $(p<.001)$.

Tabla 4. Relación entre la edad y la autonomía personal referente a las AIVD.

\begin{tabular}{|c|c|c|c|c|c|c|c|c|c|c|c|c|c|c|c|}
\hline \multirow[t]{4}{*}{ CONDUCTA } & \multirow{4}{*}{$\begin{array}{l}\text { Chi- } \\
\text { cua- } \\
\text { dra- } \\
\text { do }\end{array}$} & \multirow{4}{*}{$\begin{array}{l}\mathrm{Va}- \\
\text { lor } \\
\text { coef. } \\
\text { cont. }\end{array}$} & \multirow[t]{4}{*}{ Sig } & \multicolumn{12}{|c|}{ Residuos tipificados corregidos } \\
\hline & & & & \multicolumn{12}{|c|}{ Nivel de capacidad por edad } \\
\hline & & & & \multicolumn{3}{|c|}{ 60-69 años } & \multicolumn{3}{|c|}{ 70-79 años } & \multicolumn{3}{|c|}{ 80-89 años } & \multicolumn{3}{|c|}{ +90 años } \\
\hline & & & & S & $\mathrm{D}$ & $\mathrm{N}$ & S & $\mathrm{D}$ & $\mathrm{N}$ & $\mathrm{S}$ & $\mathrm{D}$ & $\mathrm{N}$ & S & $\mathrm{D}$ & $\mathrm{N}$ \\
\hline Cocinar & .024 & .176 & .024 & -.2 & 1.4 & -1.0 & 1.7 & .4 & -1.9 & -.3 & -1.3 & 1.4 & -2.6 & -.5 & 2.9 \\
\hline Comprar & .012 & .210 & .012 & 1.8 & .0 & -1.5 & .6 & 1.1 & -1.4 & -.8 & -.2 & .8 & -2.6 & -1.9 & 3.8 \\
\hline Limpiar & .159 & .141 & .159 & -1.5 & .7 & -.1 & 2.1 & .9 & -1.6 & -.7 & -.7 & .9 & -.8 & -1.7 & 1.9 \\
\hline Lavar/planchar & .028 & .175 & .028 & -1.5 & .2 & .7 & 3.4 & .2 & -2.2 & -1.7 & .2 & .9 & -1.6 & -1.0 & 1.8 \\
\hline Tomar medicamentos & .001 & .218 & .001 & 2.5 & -.2 & -2.6 & 1.9 & -1.6 & -1.0 & -2.3 & 1.3 & 1.7 & -3.3 & 1.1 & 2.9 \\
\hline Llevar contabilidad & .018 & .207 & .018 & 1.1 & .9 & -1.8 & 1.5 & .4 & -1.8 & -.9 & -1.0 & 1.6 & -3.0 & -.4 & 3.3 \\
\hline Usar teléfono & .000 & .279 & .000 & 2.7 & 1.0 & -3.6 & 2.2 & -2.7 & -.5 & -2.5 & 1.6 & 1.5 & -3.8 & 1.1 & 3.4 \\
\hline Tras. público & .005 & .240 & .000 & 2.9 & -.2 & -2.3 & -.8 & 2.0 & -.9 & .0 & -1.3 & 1.0 & -2.8 & -1.3 & 3.3 \\
\hline Subir/bajar escaleras & .000 & .234 & .000 & 2.7 & -1.0 & -1.3 & .9 & 2.2 & -2.8 & -1.9 & -1.0 & 2.5 & -2.5 & -1.1 & 3.2 \\
\hline Andar/pasear & .017 & .207 & .017 & 2.0 & -1.3 & -.7 & .6 & 1.0 & -1.8 & -1.1 & .4 & .7 & -2.3 & -.8 & 3.3 \\
\hline
\end{tabular}

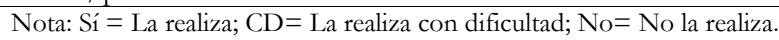

$\mathrm{Al}$ interpretar el significado de la asociación detectada, se han obtenido resultados que permiten concluir que en las variables mencionadas, son las personas mayores de 90 años donde se encuentran menos sujetos de los esperados en conductas como: cocinar, comprar, tomar medicamentos, llevar contabilidad, usar teléfono, transporte público, subir/bajar escaleras, andar/pasear. Por contra, se hallan más sujetos de los esperados entre las personas con 60 y 69 años que tienen autonomía en las siguientes conductas: tomar medicamentos, usar teléfono, uso transporte público, subir/bajar escaleras y andar/pasear.
El segundo objetivo que se planteó fue "conocer la relación entre el estado mental de los sujetos de la muestra y el factor de autonomía referente a las AIVD"

Se pretende determinar, por lo tanto, si existe relación entre estas dos variables, y si además esta relación es de dependencia, o sea, que los sujetos con menor deterioro cognitivo tienen mayor autonomía personal en la realización de las AIVD, y al contrario.

La significación de $p<.001$ permitió rechazar la hipótesis de independencia. Por lo tanto se puede afirmar que existe relación entre el deterioro cognitivo y la autonomía personal correspondiente a la ejecución de las AIVD.

Tabla 5. Relación entre el estado mental y el factor de autonomía referente a las AIVD.

\begin{tabular}{|c|c|c|c|c|c|c|c|c|c|c|c|c|c|c|c|}
\hline \multirow[t]{4}{*}{ CONDUCTA } & \multirow{4}{*}{$\begin{array}{l}\text { Chi- } \\
\text { cuadrado }\end{array}$} & \multirow{4}{*}{$\begin{array}{l}\text { Valor } \\
\text { coef. } \\
\text { cont. }\end{array}$} & \multirow[t]{4}{*}{ Sig } & \multicolumn{12}{|c|}{ Residuos tipificados corregidos } \\
\hline & & & & \multicolumn{12}{|c|}{ Nivel de deterioro según SPMSQ } \\
\hline & & & & \multicolumn{4}{|c|}{ Intacto $(0-2)$} & \multicolumn{3}{|c|}{ D. Leve (3-4) } & \multicolumn{3}{|c|}{ D. Moderado (5-7) } & \multicolumn{2}{|c|}{ D. Severo $(8-10)$} \\
\hline & & & & $\mathrm{S}$ & $\mathrm{D}$ & $\mathrm{N}$ & $\mathrm{S}$ & $\mathrm{D}$ & $\mathrm{N}$ & $\mathrm{S}$ & $\mathrm{D}$ & $\mathrm{N}$ & $\mathrm{S}$ & $\mathrm{D}$ & $\mathrm{N}$ \\
\hline Cocinar & .000 & .367 & .000 & 5.2 & 1.7 & -6.2 & -1.4 & 1.7 & -.3 & -2.6 & -1.6 & 3.8 & -3.9 & -3.2 & 6.4 \\
\hline Comprar & .000 & .318 & .000 & 3.7 & 2.4 & -5.2 & -.6 & .5 & .1 & -1.8 & -1.3 & 2.6 & -3.4 & -3.2 & 5.6 \\
\hline Limpiar & .014 & .184 & .000 & 2.1 & .7 & -1.4 & -.5 & 2.1 & -1.8 & -1.5 & -1.0 & 1.6 & -.9 & -2.5 & 2.8 \\
\hline Lavar/planchar & .000 & .254 & .000 & 2.2 & 1.7 & -2.8 & -.4 & 2.3 & -1.8 & -1.1 & -2.4 & 2.8 & -1.8 & -2.8 & 3.6 \\
\hline $\begin{array}{l}\text { Tomar medicamen- } \\
\text { tos }\end{array}$ & .000 & .500 & .000 & 8.2 & -2.6 & -7.3 & .7 & .6 & -1.1 & -5.0 & 3.6 & 3.2 & -8.5 & -1.3 & 10.2 \\
\hline Llevar contabilidad & .000 & .459 & .000 & 7.9 & .5 & -8.5 & -.7 & .8 & .1 & -5.0 & -.1 & 5.2 & -6.4 & -1.9 & 7.9 \\
\hline Usar teléfono & .000 & .480 & .000 & 8.9 & -3.4 & -7.2 & -1.4 & 2.6 & -.3 & -5.1 & -2.6 & 3.6 & -6.9 & -1.1 & 8.2 \\
\hline Tras. público & .000 & .344 & .000 & 5.5 & 1.1 & -5.5 & -.9 & .9 & .1 & -3.8 & -.9 & 3.9 & -3.2 & -1.8 & 4.1 \\
\hline Subir/bajar escaleras & .000 & .247 & .000 & 2.3 & 2.1 & -4.0 & .1 & .2 & -.3 & -1.9 & -.7 & 2.3 & -1.7 & -3.0 & 4.3 \\
\hline Andar/pasear & .000 & .297 & .000 & 2.4 & 1.8 & -4.5 & -.2 & -.3 & .6 & -1.1 & -.2 & 1.4 & -2.5 & -2.5 & 5.5 \\
\hline
\end{tabular}

Nota: $S i ́$ = La realiza; $C D=$ La realiza con dificultad; $N o=$ No la realiza. 
Como se puede observar en la Tabla 5, todas las conductas que forman parte de la autonomía personal correspondiente a las AIVD están relacionadas con el deterioro cognitivo de las personas que forman parte de la muestra, como indica el nivel de significación $p<.001$ de la prueba de chicuadrado, observando las puntuaciones que se obtienen en el coeficiente de contingencia, prácticamente todas las conductas tienen una alta asociación con deterioro cognitivo. Atendiendo a los residuos tipificados corregidos se puede apreciar como a un menor deterioro aparecen más sujetos de los esperados que pueden realizar esas conductas sin problema. Un ejemplo de ello, podrían ser las personas sin deterioro cognitivo, en las que se hallan más personas de las esperadas capacitadas para realizar sin problemas actividades como usar el teléfono $(Z=8.9)$; tomar medicamentos $(Z=$ 8.2 ), etc. frente a los sujetos con un deterioro severo, en los que en se encuentran un número inferior al esperado de personas que pueden realizar las conductas sin problemas (e. g. tomar medicamentos, $Z=-8.5$; uso del teléfono, $Z=-6.9$, etc.) por tanto, un número mayor al esperado de personas que no pueden realizar esas tareas (e. g. tomar medicamentos, $Z=10.2)$.

La respuesta dada a algunas de las conductas de índice alto de cohesión se pueden observar en las Figuras 1, 2 y 3. Estas figuras reflejan como cuando no existe deterioro cognitivo se realiza la actividad y cuando el deterioro cognitivo va aumentando, disminuye el número de personas que son capaces de realizar la actividad y crece el número de personas que no tienen autonomía suficiente para realizarlas.

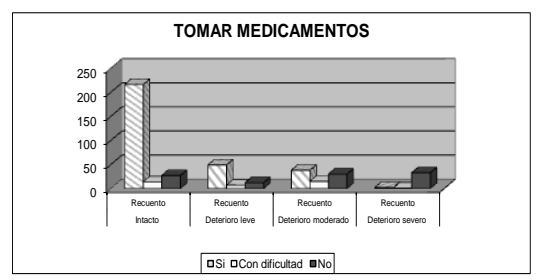

Figura 1. Relación entre deterioro cognitivo y autonomía para tomar medicación.

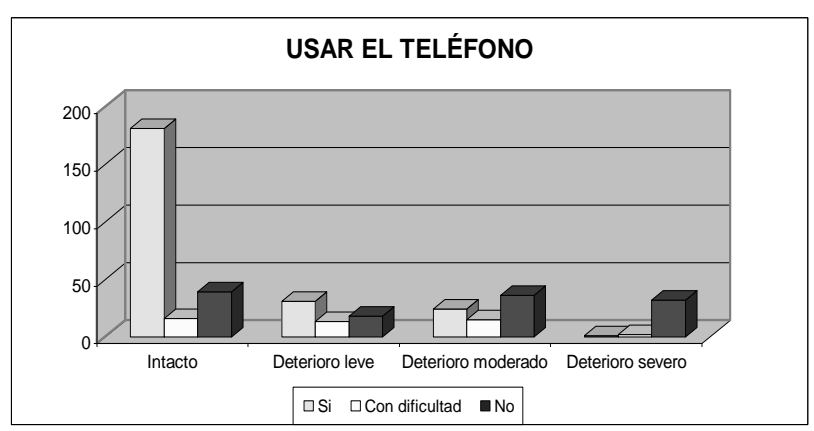

Figura 2. Relación entre deterioro cognitivo y autonomía para usar el teléfono.

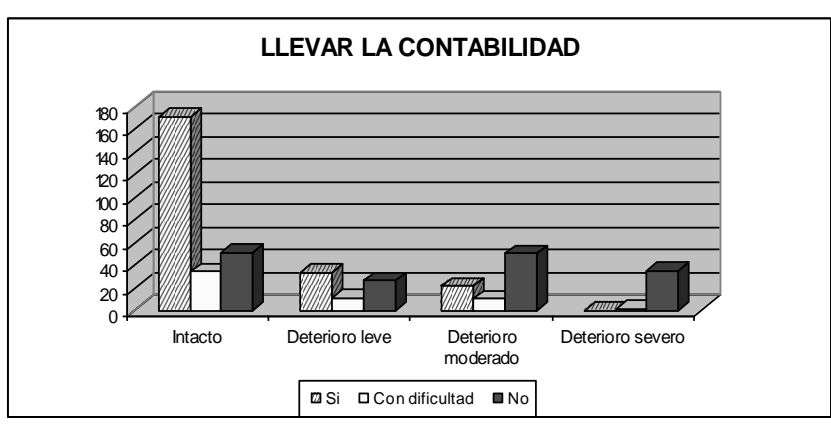

Figura 3. Relación entre deterioro cognitivo y autonomía para llevar su contabilidad.

Los resultados permiten concluir que en todas las variables de la conducta personal referente a las AIVD hay más sujetos de los esperados entre los sujetos sin deterioro y hay menos de los esperados entre los que tiene un deterioro cognitivo moderado y los que tienen un deterioro severo; salvo en la variable conductual de limpiar, en la que las diferencias entre los diferentes niveles de deterioro no son tan evidentes.

\section{Discusión}

La discusión de los resultados obtenidos hay que enmarcarlos, necesariamente, en los objetivos que se plantean para la presente investigación.

Con respecto al primer objetivo se trata de conocer qué componentes de la autonomía personal referente a las AIVD forman parte del repertorio de los usuarios de la muestra y comprobar si existen diferencias en función del sexo y edad.

En primer lugar, se focaliza la atención sobre qué componentes de la autonomía personal referida a las AIVD forman parte del repertorio de los usuarios de la muestra.

Analizando los datos obtenidos, se puede afirmar que hay un porcentaje muy amplio de sujetos que necesitan ayuda para aquellas actividades que son de tipo doméstico que se realizan a diario, sobre todo en lo referente a la limpieza de la casa y lavar y planchar la ropa.

En segundo lugar, hay que decir que existe relación entre sexo y autonomía personal correspondiente a las AIVD, siendo las conductas: cocinar, comprar, limpiar, tomar medicamentos, llevar la contabilidad, usar el teléfono, usar el transporte público, subir y bajar escaleras y andar/pasear, donde hay más hombres de los esperados que mujeres que pueden llevar a cabo estas conductas. La única conducta o actividad que desarrollan en mayor porcentaje las mujeres es lavar/planchar, que puede deberse a que es una actividad fuertemente asociadas al rol femenino tradicional, puesto que su no realización por los hombres puede no deberse a razones de incapacidad funcional adquirida sino al desconocimiento asociado a la variable género masculino.

En tercer lugar, se pretendía comprobar si también existían diferencias en función de la edad a la hora de realizar es- 
tas actividades de autonomía personal correspondiente a la realización de las AIVD.

Se puede señalar que todas las conductas evaluadas están relacionadas con la variable edad, con una significación de $p$ $<$.001. Por lo tanto, la edad sí condiciona el grado de autonomía referente a las AIVD, planteando que en las conductas evaluadas los sujetos que presentan menor autonomía son los mayores de 90 años, resaltando que en estas conductas los que tienen mayor autonomía son los de 60-69 años. Resultados coincidentes con algunas de las investigaciones que preceden a esta en las que se señala que el riesgo de padecer deterioro cognitivo y funcional aumenta de forma proporcional a la edad, como la de Atkinson et al. (2005); Gönen, Küçükgüçlü y Yener (2010); Maaskant, Akker, Kessels y Haveman (2000); Park et al. (2003); Paúl et al. (2010).

Finalmente con respecto al tercer objetivo, se observa que existe relación entre el deterioro cognitivo y el grado de autonomía personal vinculada a las AIVD. En general, se puede afirmar que los sujetos con mayor deterioro son los que menos autonomía tienen. Coincidiendo así con los resultados obtenidos por Atkinson et al. (2005) que señalaban que el deterioro físico asociado al deterioro cognitivo, provoca una disminución de capacidad para desarrollar las actividades instrumentales de la vida diaria (AIVD). Asimismo, apuntan en la misma línea que los resultados derivados del estudio de Wadley et al. (2007) que demuestran la importancia del deterioro cognitivo como una entidad clínica que no sólo predice la progresión a la demencia, sino que también predice la disminución funcional en las actividades que son clave para la autonomía y la calidad de vida de la persona mayor.

Los resultados obtenidos han permitido realizar una aproximación al conocimiento de una realidad compleja, como son las variables estudiadas, de las que se resaltan una serie de conclusiones básicas:

1. La mitad de la población, en algunos casos más, necesita ayuda para realizar las actividades propias de tipo doméstico como cocinar, limpiar la casa, lavar y planchar la ropa, etc.

2. Todas las conductas, de las que se consideran que proporcionan al mayor un estado de autonomía en la ejecu-

\section{Referencias}

Álvarez, J. y Sicilia, M. (2007). Deterioro cognitivo y autonomía personal básica en personas mayores. Anales de Psicología, 23, 272 -281.

Arthanat, S., Nochajski, S. M. y Stone, J. (2004). The international classification of functioning, disability and health and its application to cognitive disorders. Disability and Rebabilitation, 26, 235-245.

Baltes, M.M., y Werner-Wahl, H. (1990). Dependencia en los ancianos. En Carstensen, L. L. y Edelstein, B. A. Gerontología clínica. Intervención psicológica. Barcelona: Martínez Roca.

Baltes, M., Mavr, V., Borchelt, M., Maas I., y Wilms H. (1993). Everyday Competente in Old and very Old Age: an Interdisiplinary Perspectiva. Ageing and Society, 13, 657-680.

Bernal, M. A. y Barbero, M. I. (2006). Assessment of the funtional autonomy level and cognitive impairment of elderly people from a nursing ción de las AIVD, están relacionadas con el deterioro cognitivo.

Las consecuencias de la falta de autonomía en lo que se denominan "conductas de autonomía personal referentes a las AIVD" ocasiona efectos como:

a) Dependencia alimentaria, desnutrición.

b) Trastorno del ánimo, depresión, ansiedad.

c) Sentimiento de invalidez e impotencia.

d) Trastorno de la dinámica familiar.

e) Irritabilidad.

f) Baja autoestima.

g) Dependiente en trámites económicos y documentos escritos.

h) Mayor labilidad emocional.

i) Sentimientos de inutilidad.

j) Alteración del sueño.

k) Riesgos de caída

Y dado que la sociedad actual ensalza, valora y promociona la autonomía, los datos aquí presentados deben ayudar a preparar la situación para que el mayor sea de forma más fácil un ser autónomo, actuando sobre aquellas actividades sobre las que tienen más dificultad para realizarlas de forma independiente y actuando sobre las edades en las que el deterioro cognitivo dificulta en un mayor porcentaje la ejecución de las mismas.

A partir de estos nuevos conocimientos que ha proporcionado esta investigación se puede definir con más precisión qué necesidades reales demanda la población futura en cuanto a servicios sociales comunitarios, pudiéndolos tomar con la intención de optimizar y ampliar dichos servicios.

Agradecimientos.- El autor desea expresar su agradecimiento a la Consejería de Economía, Innovación y Ciencia de la Junta de Andalucía, España, por financiar este trabajo a través del Programa "Formación de personal docente e investigador predoctoral en las Universidades Andaluzas, en áreas de conocimiento deficitarias por necesidades docentes (FPDU 2008)". Dicho programa está cofinanciado por la Unión Europea a través del programa European Regional Development Fund (ERDF).

home.

Black, S. A. y Rush, R. D. (2002). Cognitive and functional decline in adult aged 75 and older. Journal of the American Geriatric Society, 50, 1978-1986.

Bouffard, L. y Bastin, E. (1994). La perspective future des persones agées fonction de la perte d'autonomie et du type d'habitation. Journal International de Pshychologie, 29, 39-53.

Bowling, A. (1994). La medida de la salud. Revisión de las escalas de medida de la calidad de la vida. Barcelona: SG-Masson.

Calero, M.D. y Navarro E. (2003). Test de posiciones: un instrumento de medida de la plasticidad cognitiva en el anciano con deterioro cognitivo leve. Revista de Neurologia, 36, 619-624.

Fernández-Ballesteros, R. (1994). Evaluación Conductual Hoy: Un enfoque para el cambio en la psicología clínica y de la salud. Madrid: Ediciones Pirámide. 
Fernández-Ballesteros, R. (1992). Mitos y realidades sobre la vejezy la salud. Barcelona: SG Editores.

Fontán, L.E., Lorenzo, J., y Silveira A. (2004). Perfil de alteración en el MiniMental State Examination en pacientes con deterioro cognitivo leve. Revista de Neurología, 39, 316-321.

Gönen, S., Küçükgüçlü, O. y Yener, G. (2010). Investigating the prevalence of cognitive and functional impairment which may be associated with dementia in hospitalized elderly. Journal of Neurological Sciences, 27(4), 446456.

Jorm, A. y Jolley, D. (1998). The incidente of dementia: a meta-analysis. Neurology, 51, 728-733.

Maaskant, M. A., Akker, M., Kessels, A. y Haveman, M. J. (2000). Care Dependence and activities of daily living in relation to ageing. Results of a longitudinal study. Journal of Intelectual Disability Research.

Neugarten, B.L. (1982). Age or Need? Public Policy for older people. Beverly Hills: Sage.

Paúl, C., Ribeiro, O. y Santos, P. (2010). Cognitive impairment in old people living in the community. Archives of Gerontology and Geriatrics, 51(2), 121124.

Park, H., O’Connell, J. y Thomson, R. (2003). A systematic review of cognitive decline in the general elderly population. International Journal of Geriatric Psychiatry, 18, 1121-1134.
Pfeiffer, E. (1975). A Short portable mental status questionnaire. For the assessment of organic bain deficit in ederly patients. Journal of the American Geriatric Society, 23, 433-441.

Schulz, R., Heckhausen, J. y O’Brien, (1994). Control and the Disablemnent Process in the Elderly. Journal of Social Behavior and personality, 9, 139-152.

Ser Quijano, T. Del y Peña-Casanova, J. (1994). Evaluación neuropsicológica y funcional de la demencia. Barcelona: J. R. Pruos Editores.

St John, P.D., Montgomery, P.R., Kristjansson, B. y McDowell, I. (2002). Cognitive scores, even within the normal range, predict death and institutionalization. Age Ageing, 31, 373-378.

Wadley, V.G., Crowe, M., Marsiske, M., Cook, S.E., Unverzagt, F.W., Rosenberg, A.L. y Rexroth, D. (2007). Changes in everyday function in individuals with psychometrically defined mild cognitive impairment in the advanced cognitive training for independent and vital elderly study. Journal of the American Geriatrics Society 55, 1192-1198

Werner, P. y Korczyn, A. D. (2008). Mild cognitive impairment: Conceptual, assessment, ethical, and social issues. Clinical Interventions in Aging, 3(3), 413-420.

Whechsler, D. (1945). A Standardized memory scalefor clinical use. Journal of Psychology, 19, 87-95.

(Artículo recibido: 24-11-2009, revisión: 24-9-2011, aceptado: 4-12-2011) 